\title{
Computed tomography in diagnosis and staging of the retroperitoneal tumors
}

${ }^{1}$ Faculty of Medicine, University "Ovidius" of Constanta

\begin{abstract}
The main role of retroperitoneal tumor diagnosis is the imaging examination. Diagnostic imaging is essential for describing and predicting lesion integrity limits adjacent organs.

Methods. The results are based on a sample of 53 patients admitted in the Urology Clinic and investigated during the period 1 January 2005 - 31 December 2014. Results. CT scan for retroperitoneal tumor: vascular tumors are diagnosed in $100 \%$ of cases, while endocrine tumors and lymphomas values are between 50-66.67\% in terms of a positive diagnosis. In $54.71 \%$ of cases studied was identified retroperitoneal tumor invasion into adjacent structures (29 patients), and for 12 cases (22.64\%) CT scan was not conclusive enough. CT scan investigation to identify metastasis has statistical significance $(p<0.01)$ according to the different types of retroperitoneal tumors, there were on average $35 \%$ for highlighting values of peritumoral lymph nodes and $13 \%$ for metastases. The relationship between retroperitoneal tumor size detected by CT (coefficient R $=0.736$ ) than those found intraoperative $\mathrm{CT}$ examination reveals that tends to underestimate the retroperitoneal tumor size

Conclusions. Imaging diagnosis is mainframe of positive diagnosis for retroperitoneal tumors. CT scanning bring data not only on tumor location, but also
\end{abstract}

Osman Rauf

Faculty of Medicine, University "Ovidius" of Constanta

University Street, No. 1, Campus B

Constanta, Romania about the nature of injuries (cystic or solid), presence or absence of necrosis, invasion of adjacent structures and abdominal metastases.

Keywords: imaging diagnosis, retroperitoneal tumor

\section{Introduction}

Retroperitoneal tumors consist of a heterogeneous group of clinico-pathological entity, the most frequent being carcinoma metastasis.

The anatomic space known as retroperitoneal, a territory of anyone or everyone, gives meeting endocrinologists, vascular or general surgeons, , urologist, neurosurgeons and orthopedics, which is due to organs situated wholly or partly here or vascular elements, nerves, embryonic or ectopic tissue "wrapped" in a rich adipose and connective tissue [1].

Retroperitoneal space includes adrenal glands, kidneys and ureters, aorta and its branches, the inferior vena cava and its tributaries, as well as numerous lymph nodes and paraganglions, all these structures are embedded plentifully in a soft connective tissue. By convention, the name of primitive retroperitoneal tumor (TRP) is provided to those tumors developed in this space, outside the cited structures. Primitive or secondary (metastatic), retroperitoneal tumors usually grow silently before clinical signs and symptoms due to compression or obstructive phenomena manifest $[2,3]$.

From a morphological classification, it 
describes four categories of retroperitoneal tumors: tumors of soft tissues, malignant lymphomas, germ cell tumors and secondary/ metastatic tumors. Although their evolution and prognosis are influenced by histological variability of these neoplasms, they have many similar features, the therapeutic approach is most often independent of histologic origin.

Due to poor symptoms the imaging explorations have a crucial role in the diagnosis of retroperitoneal tumors. Diagnostic imaging is essential for describing lesion borders and predicting the integrity of nearby organs [4].

Given the wide diversity of signs and symptoms that a retroperitoneal tumor especially malignant can take, to an early diagnosis it is extremely important to know all the symptoms of tumor pathology, ways of onset and incidence of each such opportunities in practice. The finding in very early stage of retroperitoneal tumors, without clinical manifestations, is an essential condition for an effective therapeutic intervention and a favorable prognosis.

\section{Methods}

This study is realized in Constanta County Emergency Hospital and includes case studies of Urology and Surgery Clinics during 2005-2014.

Results are based on a sample of 53 patients admitted and investigated in the clinics during the period 1 January 2005 - 31 December 2014.

Retroperitoneal tumors diagnosis was based on clinical data (history, physical examination) and laboratory data (less the laboratory and in particular imaging - ultrasonography, computed tomography, magnetic resonance, X-ray, angiography). For a proper diagnosis must follow several steps, carrying her to omit one or superficiality can lead to a wrong diagnosis, prognosis and could cause considerable prejudice.

The cases studied were investigated in terms of radiological and imaging using abdominal ultrasound, axial computed tomography $\mathrm{CT}$ with and without contrast agent, simple abdominal X-ray and intravenous urography, nuclear magnetic resonance MRI and angiography for a smaller number of cases.

\section{Results}

If in the early years for cases hospitalized in SCJU Constanta abdominal ultrasound was the method of choice for imaging diagnosis, in subsequent years could be observed shifting preferences by abdominal CT scan, which became mandatory in such cases, MRI and subsequent angiography bringing additional data about the extension of tumor. Sonography was performed and prior hospitalization, it sometimes leading to suspicion of renal tumor and the first stage of diagnosis. Angiography and MRI are represented in low percentage, because of high costs on investigation and reserved only in cases of uncertain diagnosis (Table I).

Table I - Imagistic investigation

\begin{tabular}{|l|c|c|c|}
\hline Method & $\begin{array}{l}\text { No. of } \\
\text { cases }\end{array}$ & $\begin{array}{l}\text { Percent } \\
\%\end{array}$ & $\begin{array}{l}\text { Confidence } \\
\text { interval 95\% }\end{array}$ \\
\hline Ultrasonography & 51 & 96.22 & $93.2 \%-99.8 \%$ \\
\hline CT scan & 53 & 100 & $95 \%-100 \%$ \\
\hline $\begin{array}{l}\text { X-ray/ } \\
\text { Urography }\end{array}$ & 19 & 35.85 & $30.85 \%-40.85 \%$ \\
\hline MRI & 23 & 43.39 & $38.4 \%-48.4 \%$ \\
\hline Angiography & 3 & 5.66 & $1,1-9,6 \%$ \\
\hline
\end{tabular}

CT scan in association to ultrasound examination appears to be valuable in essential preoperative positive diagnosis in TRP.

CT was performed in all patients in the study group: vascular tumors are diagnosed at a rate of $100 \%$, while endocrine tumors and lymphomas recorded values between $50-66.67 \%$ concerning positive diagnosis (Figure 1). With a high rate of preoperative diagnosis enroll sarcomas, lipomas and nerve sheath tumors with ultrasound appearance of 
doubt (cysts, teratomas).

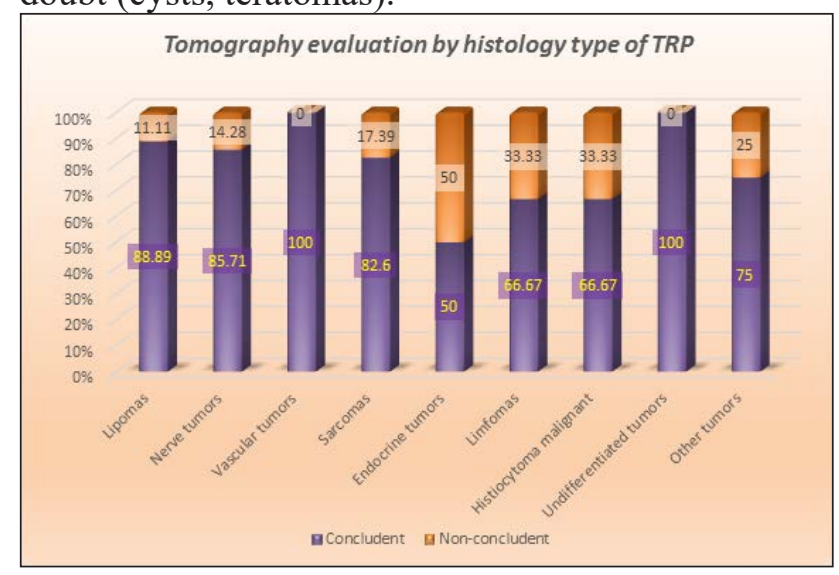

Figure 1 - Tomography evaluation by histology type of TRP

From the statistical point of view this is statistically significant finding ( $p=0.011$, Chi square test) which lends credibility to imaging methods considered in this way.

It should be noted that the larger diameters of the retroperitoneal tumors are sarcomas $(16.75 \mathrm{~cm})$, malignant fibrous histiocytoma $(13.65 \mathrm{~cm})$, whereas vascular and endocrine tumors have the lowest size. Even if there are large differences between the average diameters of studied tumor types, statistical results do not reach the threshold of statistical significance $(\mathrm{p}=0.21$, Chi square test $)$ probably due to the small number of cases (Figure 2).

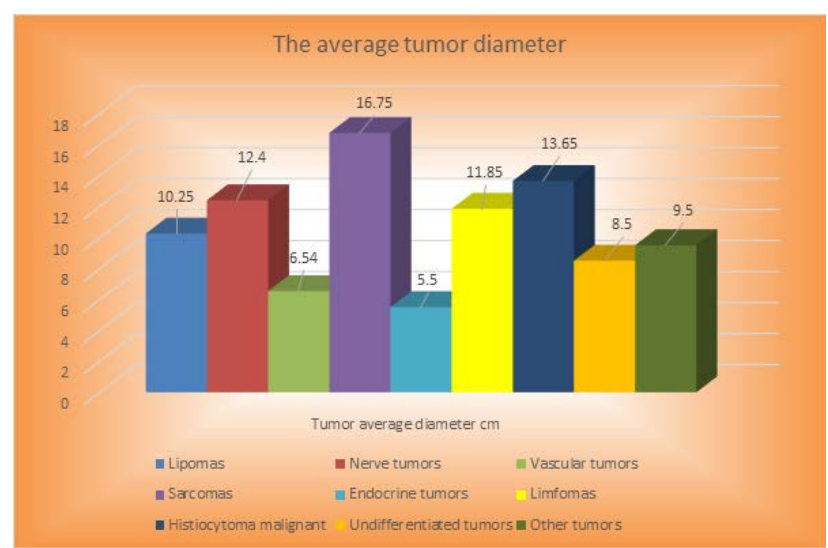

Figure 2 - Average tumor diameter (CT scan) according to histopathological tumor types

Primitive retroperitoneal tumors show variable density (Hounsfield Units), it depends on the presence of necrotic tissue, hemorrhage or calcification. Although outlines can often be appreciated, there may be small tumors not distinguished without contrast agent [5].

Just as important characteristic appearance for TRP histopathological structure is assessing of loco regional invasiveness (Figure 3).

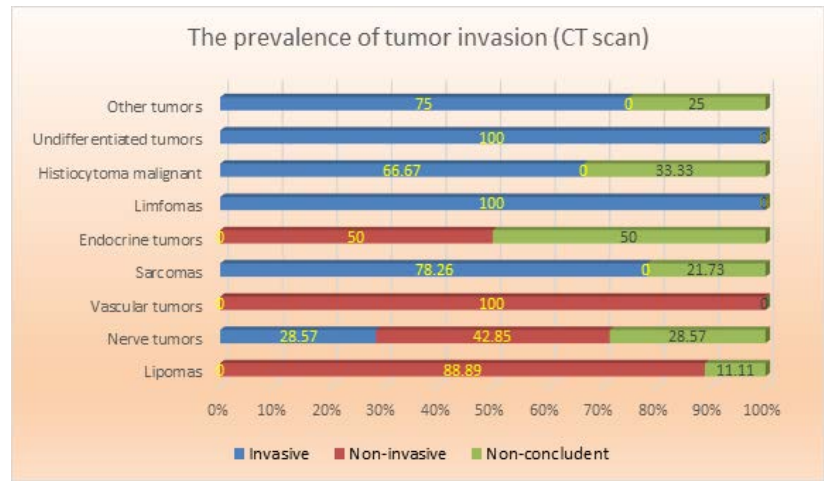

Figure 3 - The prevalence of tumor invasion (CT) according to histopathological tumor types

Perirenal space - poor tumor delineation and of hyper-dense trabecular extensions in retroperitoneal fat are considered specific for tumor invasion. In thin subjects, the low amount of perirenal and retroperitoneal fat may suggest a tumor involution while a large neoplasm may compress kidney fat.

Perirenal space and other organs - tissue invasion associated with extension to retroperitoneal fascia Gerota is a frequent, can identify the direct invasion of neighboring organs, for example the image of a tumor is prolonged to another organ with a heterogeneous density area or there is a continuous area of neovascularization using a contrast agent [6].

Given the fact that more than half $(54.71 \%)$ of the cases studied has identified the presence of retroperitoneal tumor invasion into adjacent structures (29 cases) and CT was not sufficiently conclusive in 12 cases $(22.64 \%)$, may note that a high percentage of patients had tumor invasion in malignant types.

Vascular venous extension - assessing vascular extension is crucial in evaluating of TRP, there are a large number of views on the CT versus other imaging methods, the study the renal veins requiring special procedures - angioscan. Compression and 
thrombosis of the inferior vena cava or renal vein induce changes in morphology and vessel density: more than $1.5 \mathrm{~cm}$ in diameter is a diagnostic sign when it is combined with density fluctuations that occur as inhomogeneous filling defects with or without contrast agent (10-20 HU) (Figure 4). Some aspects may be omitted: intensely vascularized neoplastic thrombi can have high density (100 HU) which mimics blood flow. In these cases, angioscan may be helpful by simultaneous comparison with $\mathrm{CT}$ scan [7].

In sagittal and frontal reconstruction vena cava is easier to appreciate, the shape and diameter may change with respiration from 1.5 to $3.7 \mathrm{~cm}$. The vena cava status can be pursued also by injecting a contrast agent into the veins of the lower leg. Vena cava thrombosis should be suspected in the presence of large tumors extended to the renal hilum and furthermore associated with renal vein thrombosis. The diagnosis is confirmed by the following criteria: over $3.7 \mathrm{~cm}$ diameter, intraluminal filling defects sometimes circumscribed by a peripheral ring or halo area.

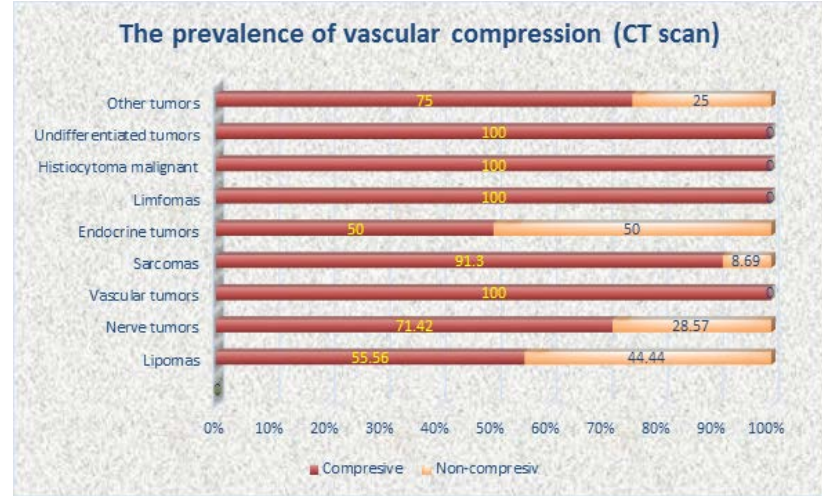

Figure 4 - The prevalence of vascular compression (CT scan) according to histopathological tumor types

Examination by CT scan is useful in distinguishing different types of clots: neoplastic thrombus has a characteristic appearance by circular, hyperdense and inhomogeneous structure, suggestive for an organized thrombus with hypervascularization.

Lymphatic extension - CT detects metastatic lymph nodes as round clusters larger than $5 \mathrm{~mm}$ in diameter with poor contrast, that are grouped around the vessels, but this exam may not provides enough data to clarify the etiology of adenopathy (there may be inflammatory lymph nodes) (Figure 5)

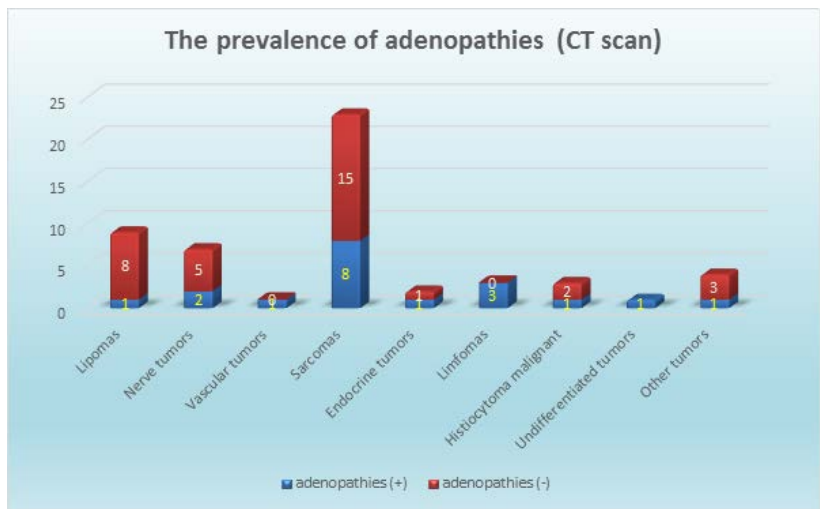

Figure 5 - The prevalence of adenopathies (CT scan) according to histopathological tumor types

CT appearance reflects pathologic model of solitary tumor, multiple nodules or diffuse injury; there can be observed lymphadenopathies and extension to other organs (Figure 6).

CT contribution is important in considering peritumoral lymph nodes and distant metastases, thus TNM staging can be complete.

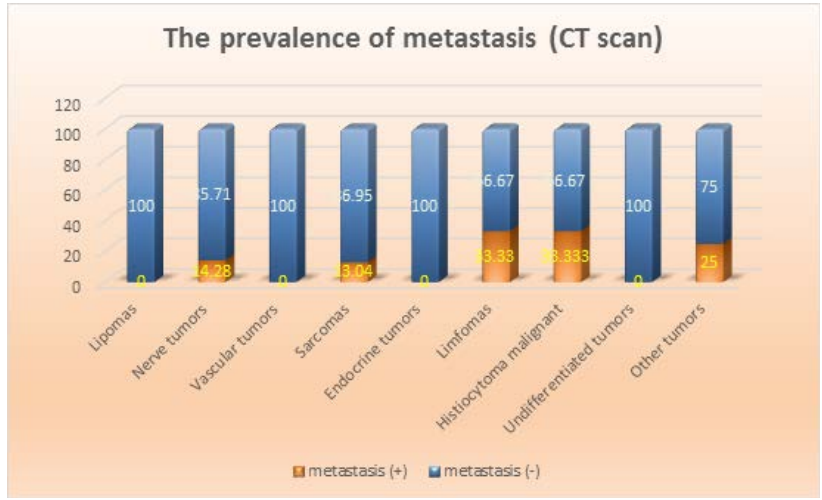

Figure 6 - The prevalence of metastasis (CT scan) according to histopathological tumor types

Statistical analysis of data presented in Tables VI and VII show that the CT results have a statistical significance $(\mathrm{p}<0.01)$ according to the different types of retroperitoneal tumors. It is observed that tumors like sarcomas, vascular tumors and histiocytomas are accompanied by a low rate of lymph node and distant metastasis. At the opposite pole are placed 
lymphomas (lymph nodes $100 \%$ evident in the CT), endocrine tumors, neurological and histopathological uncertain origin tumors. On average there were 35\% for highlighting values of peritumoral lymph nodes and $13 \%$ in the presence of distant metastases.

The most important correlation analysis of this method of imaging investigation is still assessing a tumor size detected by CT than that found intraoperative. To assess this, we proceeded to statistical analysis using correlation test and Pearson simple linear regression test (Table II).

Table II - R (Pearson) coefficient values

\begin{tabular}{|l|c|c|c|}
\hline Statistical data & Average & $\begin{array}{c}\text { Standard } \\
\text { deviation }\end{array}$ & $\begin{array}{c}\text { No. of } \\
\text { cases }\end{array}$ \\
\hline CT - tumor diameter & 10.54 & 3.53 & 53 \\
\hline $\begin{array}{l}\text { Diameter during } \\
\text { surgery }\end{array}$ & 13.55 & 5.07 & 53 \\
\hline R coefficient & \multicolumn{3}{|c|}{0.736} \\
\hline
\end{tabular}

Obviously the two dimensions measured are strongly correlated, as demonstrated by the coefficient $\mathrm{R}=(+) 0736$.

A linear correlation shows that $\mathrm{CT}$ examination tends to underestimate the retroperitoneal tumor size. Thus, the average size of tumors are measured by the CT average of $73.6 \%$ of the size of the measured during surgery. In other words retroperitoneal tumors identified by abdominal CT are larger than those found on CT films.

Additional statistical tests (Table III) shows that the relationship is valid regardless of tumor size, CT examination underestimates both the large and small tumors, not often this relationship has no statistical explanation.

Table III - ANOVA analysis - correlation between tumor size (CT scan and intraoperative)

\begin{tabular}{|l|c|c|c|}
\hline Analysis & Regression & Residual & Total \\
\hline Square sum & 1895.5 & 1101.6 & 2997.1 \\
\hline $\begin{array}{l}\text { Degrees of } \\
\text { freedom }\end{array}$ & 1 & 53 & 54 \\
\hline Squares average & 1895.5 & 20.78 & - \\
\hline F test & 58.752 & & \\
\hline Significance & $<0.0001$ & & \\
\hline
\end{tabular}

The reliability of the $\mathrm{CT}$ examination is superimposable on the ultrasound exam and has the advantage of simultaneous clouding of vessels and viscera or digestive tract. Summarizing the advantages of $\mathrm{CT}$ imaging in the diagnosis of retroperitoneal tumors can retain the following:

- Data on retroperitoneal tumor origin, dimensions, relations with neighboring organs and expansion loco-regional tumor are more conclusive than the abdominal ultrasound. Not to be overlooked that TRP image by CT is on average $26.3 \%$ lower than intraoperative reality.

- Tomography provides important details about the structure of retroperitoneal tumors making it possible to identify in many cases the histological type. The imaging results are accompanied and correlated with histopathologic diagnosis on paraffin up to $100 \%$ in some cases (vascular tumors, lymphoma).

- relations with neighboring organs: compression, displacement, invasion; detecting the boundaries between the tumor and neighboring viscera indicates the absence of extension

- Tumor vessels;

- The sensitivity of CT in detecting peritumoral lymph nodes exceed $85 \%$.

- Experience presented notes the usefulness of CT in the detection of distant metastases.

- A particular advantage it has over MRI has spiral CT that provides a higher contrast of soft tissue. Calcified or increased density lesions are easily detected on CT, this is not always possible to MRI, which calcifications are viewed as filling defects, this can be determined by a stone, a tumor fragment or blood clot.

\section{Conclusions}

The rarity of primitive retroperitoneal tumors is specified for most data from medical literature and confirmed in this study. From here it arises the 
complexity of diagnostic and treatment of these cases along with the anatomical and surgical particularities.

Imaging diagnostic is core element of positive diagnosis in the TRP and rely on combination of abdominal computed tomography and abdominal ultrasonography. These investigations underlying algorithm diagnosis of primary retroperitoneal tumors, a useful diagnostic tool revealed by this study. Computerized axial tomography CT remains the most reliable and accurate method of assessment of retroperitoneal tumors, its high accuracy to be useful in the differential diagnosis of tumors. In most cases CT scan confirmed the diagnosis already suspected by other imaging methods previously used.

Computed tomography (CT) bring data not only on the location of the tumor, but also the nature of the lesions (cystic or solid), presence or absence of necrosis, invasion of adjacent structures and the presence of abdominal metastases, it represents the most important exploration imaging procedure for diagnosis and staging of retroperitoneal tumors.

\section{References}

1. Netter, F. H. (2010). Atlas of human anatomy (5 $5^{\text {th }}$ ed.). Publisher: Saunders.

2. Codoiu, C. Hăineală, B., Margaritis, S., Bădescu, B., Preda, A., Baston C. \& Sinescu, I. (2015). Dedifferentiated liposarcoma of the retroperitoneum. Revista Română de Urologie. 14(74), 73-75.

3. Vasile, I., Vîlcea, D., Nemes RCurcă. T., Pasalega M., Calotă F., Paraliov T., Mesină C. \& Mogos, D. (2006). Tumorile retroperitoneale primitive probleme de diagnostic si tratament. Chirurgia. 100(1), 27-33.

4. Buzoianu, M., Filip, F.A. \& Lupescu I.G. (2015). Retroperitoneal Cystic Masses: an overview of $C T$ and MRI findings. Poster session presented at the European Congress of Radiology

5. Morosi, C., Stacchiotti, S., Marchianò, A., Bianchi, A., Radaelli, S., Sanfilippo, R. \& Fiore,
M. (2014). Correlation between radiological assessment and histopathological diagnosis in retroperitoneal tumors: Analysis of 291 consecutive patients at a tertiary reference sarcoma center. European Journal of Surgical Oncology (EJSO). 40(12) 1662-1670.

6. Brooks, J. S. \& Lee, S. (2015). Contemporary diagnostics: Sarcoma pathology update. Journal of surgical oncology. 111(5), 513-519.

7. Cocieru, A. \& Saldinger, P. F. (2011). Images in surgery: retroperitoneal ganglioneuroma. The American Journal of Surgery. 201(1), e3-e4. 